\title{
LA PRODUCCIÓN DE CONOCIMIENTO EN LAS SOCIEDADES CONTEMPORÁNEAS
}

\author{
Rosa Judith Arias Rey \\ Asesora Metodológica IMA
}

En algunos países en vía de desarrollo existe la falsa creencia de que la Investigación Científica se debe dejar a las grandes potencias, y que, para las necesidades "reales" del país, se dispone de científicos y tecnólogos en cantidad y calidad suficientes. También se encuentra la idea de que el principal objetivo de la planificación estatal de la investigación es orientarla hacia actividades útiles, lo cual se lograría fijando prioridades por medio de la asignación selectiva de fondos. Reducir la política científica a esos términos se vería de manera no sólo simplista sino, también, equivocada. Una de las razones por las cuales se puede considerar ésta como una visión errónea es que omite concebir al conocimiento como un fin en sí y lo reduce a un mero medio para lograr otros objetivos. Por otro lado, una política científica de este tipo, excluye de los planes estratégicos la formación de recursos humanos, así como deja de lado establecer mecanismos que articulen entre ellos a los diversos actores de la labor científica, dos asuntos cuya ausencia da lugar a muchas de las causas de nuestro subdesarrollo en esta materia.

Para comprender las relaciones actuales de la ciencia y la tecnología con el crecimiento económico, debe recordarse que los procesos de producción en masa y las economías de escala, que en otra época fueron los más poderosos motores de ese crecimiento, hoy en día cedieron su función impulsora a las industrias basadas en el conocimiento. Pero

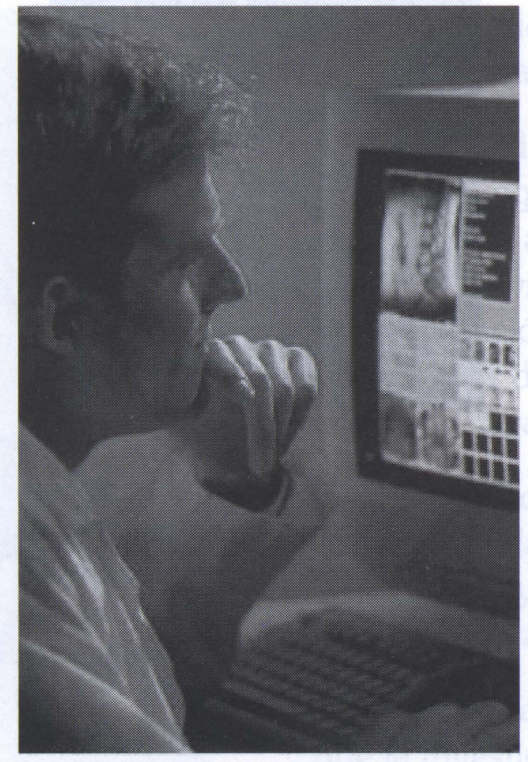

éstas sólo pueden prosperar en sociedades que estén en condiciones de acceder a la información, evaluarla, organizarla y usarla; en otras palabras, que sean capaces de utilizar conocimientos nuevos o de aprovechar los existentes para mejorar o desarrollar procesos, productos o servicios, esto es, producir innovaciones. Se puede decir que las sociedades que cuentan con estas capacidades disponen de un sistema nacional de innovación.

Dichas sociedades destinan a la ciencia y la tecnología entre el $2 \%$ y $3 \%$ del producto interno bruto, en partes aproximadamente iguales por los sectores público y privado. Para poder realizar ese esfuerzo, poseen suficientes recursos humanos capacitados y una organización que permite la articulación ágil y eficiente de los distintos responsables de la producción de ciencia y tecnología. Los recursos financieros se distribuyen en proporciones varia- bles entre los diversos modos de hacer ciencia; un patrón característico es el de países como los Estados Unidos, Canadá, Japón y los de la Unión Europea que, en promedio, destinan el $15 \%$ a financiar ciencia académica (creación de conocimiento original en cualquier rama de las ciencias, las humanidades y la tecnología), el $25 \%$ a ciencia aplicada $\mathrm{u}$ orientada (utilización de conocimientos existentes o búsqueda de nuevos para resolver problemas predeterminados) y el $60 \%$ a desarrollo tecnológico (uso de resultados de la investigación para crear productos, procesos o servicios útiles). La disparidad de las asignaciones no refleja prioridades sino los distintos costos de cada actividad. La investigación académica se financia, sobre todo, con fondos públicos, los que están presentes de modo decreciente a medida que la tarea se acerca a los productos de utilidad inmediata. Inversamente, los fondos privados van sobre todo a los desarrollos tecnológicos y en mucha menor medida a la ciencia académica.

Los tres modos de realizar ciencia son indispensables para alcanzar los máximos beneficios sociales. Está cada vez más claro que los vínculos entre ciencia básica, aplicada y desarrollo tecnológi-

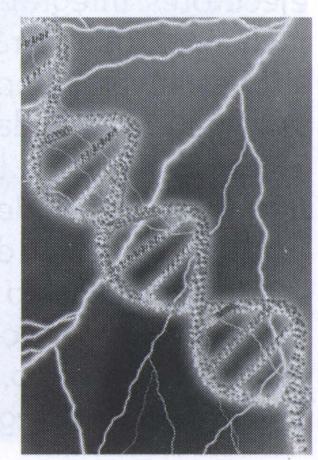


co no se ajustan a la idea, a veces sostenida por investigadores académicos, de que existe una progresión lineal y espontánea que va de la investigación básica a la aplicada y de allí al desarrollo tecnológico. Las relaciones entre esas actividades se parecen más a las imperantes en un ecosistema, donde cada componente requiere y utiliza a los restantes. Tradicionalmente, se consideraba que la ciencia académica era la principal fuente de innovaciones y de recursos humanos capacitados, punto de vista cuestionado por cierta literatura reciente, que señala la creciente importancia de las industrias basadas en el conocimiento para la competitividad de las economías. Esa literatura habla de un nuevo modo de producir conocimiento, que llama 'modo 2' para diferenciarlo del tradicional, que seria el 'modo 1'.

En el modo 1, la ciencia es monodisciplinaria y se realiza en organizaciones jerárquicas permanentes (universidades y centros de investigación) con el objetivo primario de avanzar en el conocimiento de la realidad. Sus metas son fijadas por los propios investigadores y sus productos son del dominio público y sólo se convierten en parte de una disciplina luego de un cuidadoso control por la comunidad de pares. La investigación del modo 2 es transdisciplinaria, sus ejecutores integran transitoriamente grupos no jerárquicos, que se constituyen ad hoc para resolver alguna demanda social y luego se disuelven, y la validación de sus resultados descansa en la aceptación social del producto más que en el juicio de los pares. En contraste con lo que sucede con el primer modo, los temas de investigación del segundo vienen

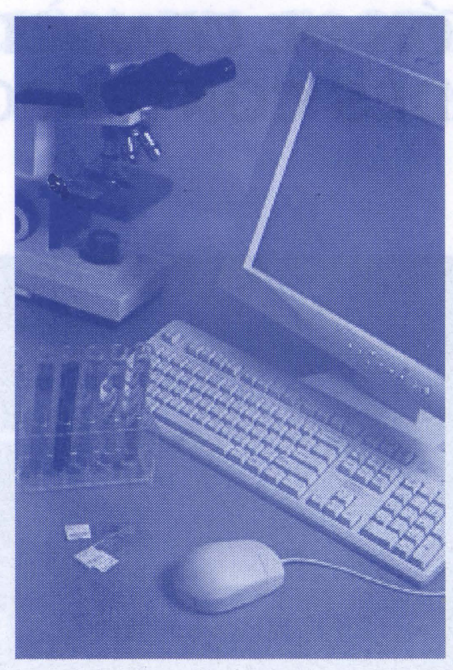

dados por las razones que dan origen al grupo de trabajo, y sus productos son de propiedad privada y están protegidos por patentes. Quizá la diferencia más substancial entre ambos modos de crear conocimiento reside en que el tradicional concentra sus esfuerzos en la búsqueda de principios generales, mientras el nuevo quita el énfasis central de tal búsqueda y lo pone en la manipulación innovadora de lo ya conocido.

Si predominara fuertemente la nueva forma descripta, cambiaría de manera radical el significado de la tarea científica, pues se pierde objetividad, ya que está condicionada por los intereses que dan origen al grupo de trabajo $y$, por lo tanto, sometida a presiones de factores ajenos a la investigación.

Más allá del juicio que pueda merecer, el modo 2 de hacer ciencia es un ejemplo extremo de cómo los países desarrollados tratan de articular los diversos tipos de ciencia entre ellos, por medio de mecanismos eficientes, y de vincular la ciencia con los otros sectores, para obtener los máximos beneficios sociales de la producción de conocimiento. Si se compara lo que acontece en esos países con Colombia, resulta que aquí el gasto público y privado en ciencia no sólo es muy bajo sino que ha permanecido constante o ha disminuido en los últimos años, sobre todo si se tienen en cuenta los costos crecientes de la investigación y el aumento del número de investigadores.

Predomina la investigación académica financiada por el sector público, hay escasa inversión en desarrollo tecnológico y, en general, se piensa en términos del modelo lineal de los vínculos entre investigación básica, investigación aplicada y desarrollo tecnológico. Con relación a la población, el número de científicos y tecnólogos de Colombia es un décimo del de los países desarrollados. Por ello no debe sorprender la escasa capacidad del país de llevar adelante investigaciones básicas o aplicadas, al igual que desarrollos tecnológicos adecuados frente a las condiciones competitivas del mundo actual.

Se requiere entonces, que las políticas públicas de ciencia y tecnología sean políticas de Estado que apunten al largo plazo, más allá de los cambiantes intereses políticos de los gobiernos, y que las reglas del juego sean claras, sin discontinuidades ni correcciones bruscas. Para que Colombia pueda acercarse en esta materia al primer mundo, el nivel del gasto público y privado en la producción de ciencia y tecnología, en términos relativos a la capacidad productiva de la economía, deberá asemejarse al de los países desarrollados. Y no hay que olvidar los procesos de formación de recursos humanos, sin los cuales será imposible incrementar el esfuerzo, ni omitir el muy importante imperativo de defender la objetividad e independencia de la ciencia académica. 